\title{
Mycoplasma genitalium: high prevalence of resistance to macrolides and frequent anorectal infection in men who have sex with men in western Sydney
}

\author{
Deborah L Couldwell, ${ }^{1,2}$ Dean Jalocon, ${ }^{3}$ Melissa Power, ${ }^{1}$ Neisha J Jeoffreys, ${ }^{3}$ \\ Sharon C-A Chen, ${ }^{2,3}$ David A Lewis ${ }^{1,2}$
}

'Western Sydney Sexual Health Centre, Parramatta, New South Wales, Australia

${ }^{2}$ Marie Bashir Institute for Infectious Diseases and Biosecurity and Sydney Medical School-Westmead, University of Sydney, Sydney, New South Wales, Australia

${ }^{3}$ Centre for Infectious Diseases and Microbiology Laboratory Services, ICPMR, NSW Health Pathology, Westmead, New South Wales, Australia

\section{Correspondence to} Dr Deborah L Couldwell, Western Sydney Sexual Health Centre, Parramatta, NSW 2150 Australia; deborah.couldwell@ health.nsw.gov.au

Received 21 November 2017 Revised 4 February 2018 Accepted 17 February 2018 Published Online First 22 March 2018

\section{Linked}

- http://dx.doi.org/10.1136/ sextrans-2017-053367

- http://dx.doi.org/10.1136/ sextrans-2017-053376

Check for updates

To cite: Couldwell DL, Jalocon D, Power M, et al. Sex Transm Infect 2018:94:406-410.

\begin{abstract}
Objectives We aimed to estimate the prevalence of Mycoplasma genitalium infection and of mutations linked to macrolide resistance using the ResistancePlus MG assay (SpeeDx, Sydney, New South Wales, Australia) in first-void urine (FVU), anorectal and oropharyngeal samples from men who have sex with men (MSM) attending Western Sydney Sexual Health Centre (WSSHC).
\end{abstract}

Methods Consecutive symptomatic and asymptomatic MSM attending for STI testing were prospectively enrolled. M. genitalium testing using the ResistancePlus MG assay was performed on FVU, anorectal and oropharyngeal samples routinely collected for Chlamydia trachomatis and Neisseria gonorrhoeae assays.

Results Overall, the prevalence of $M$. genitalium infection in the study group was $13.4 \%$ (68/508). Most (79.4\%, 54/68) M. genitalium harboured macrolide resistance mutations ( $87.5 \%$ of urethral and $75.6 \%$ of anorectal infections). The anorectum was the most commonly infected site $(45 / 505,8.9 \%)$, followed by the urethra $(24 / 508,4.7 \%)$. No oropharyngeal $M$. genitalium infections were detected (0/508). Most of the anorectal (93.3\%) and urethral (79.2\%) infections were asymptomatic. MSM who were taking HIV pre-exposure prophylaxis (PrEP) were twice as likely to be infected with $M$. genitalium compared with MSM who were not on PrEP (OR 2.1, 95\% Cl 1.3 to 3.6; $\mathrm{P}=0.0041$ ). Always using condoms for anal sex in the last 3 months was protective of infection (OR $0.8,95 \% \mathrm{Cl} 0.6$ to 1.0 ; $\mathrm{P}=0.0186$ )

Conclusions We demonstrated a high prevalence of $M$. genitalium and very high levels of macrolide resistance among MSM attending WSSHC. Our findings support the routine use of an assay to detect macrolide resistance mutations in M. genitalium infections. This will ensure, in regions or populations with high rates of macrolide resistance among $M$. genitalium strains, that first-line treatment with azithromycin will only be used if a macrolide-sensitive strain is identified.

\section{INTRODUCTION}

Although Mycoplasma genitalium is a well-established cause of non-gonococcal urethritis (NGU), few reports describe asymptomatic or extragenital infections in men who have sex with men (MSM), and evidence is lacking as to the value of screening for M. genitalium in asymptomatic populations. ${ }^{1-7}$ More information about the pattern of infection, clinical manifestations and biological ramifications of M. genitalium infection in MSM would inform testing guidelines. Furthermore, internationally there have been widespread reports of increasing $M$. genitalium resistance to azithromycin, a macrolide antibiotic used for first-line treatment. ${ }^{8}$ MSM populations may be particularly vulnerable to acquiring and transmitting macrolide resistant $M$. genitalium due to their higher risk of STIs and the increased likelihood of prior macrolide therapy.

From 2001 to 2014, the overall reported M. genitalium prevalence among MSM has ranged from $2.1 \%$ to $8.1 \%$ (1.6\%-5.4\% in anorectal infections), but none of these studies examined the prevalence of M. genitalium macrolide resistance. ${ }^{1-6}$ Oropharyngeal M. genitalium has rarely been reported. ${ }^{9}$

Antimicrobial resistance has complicated the treatment of M. genitalium infections. Macrolide resistance-associated mutations in region $\mathrm{V}$ of $23 \mathrm{~S}$ rRNA limit effectiveness of initial treatment with azithromycin, and fluoroquinolone resistance is impacting the effectiveness of second-line therapy, leading to ongoing transmission of resistant $M$. genitalium strains. ${ }^{10} 11$ In Australia, macrolide resistance mutations were present in $36 \%-43 \%$ of strains detected mainly in men with NGU between 2008 and 2013. ${ }^{12}$ Macrolide resistance testing when M. genitalium is detected could reduce time to cure and thereby limit ongoing transmission of macrolide-resistant strains. ${ }^{7}$

In the present study, we aimed to estimate the prevalence of M. genitalium infection as well as the prevalence of mutations linked to macrolide resistance using the ResistancePlus MG assay (SpeeDx Sydney, New South Wales, Australia) in urine, anorectal and oropharyngeal samples from MSM attending Western Sydney Sexual Health Centre (WSSHC).

\section{METHODS}

WSSHC provides comprehensive STI testing and treatment for at-risk populations including MSM, sex workers and people living with HIV infection. 
MSM accounted for approximately $50 \%$ of 8139 visits to the clinic in 2017. Individual patient consent was not required. A sample size of 500 men was calculated assuming prevalence of M. genitalium of $5 \%$, test sensitivity of 0.7 , specificity of 0.9 , at 0.95 confidence and 0.05 precision. Consecutive symptomatic and asymptomatic MSM attending the sexual health centre in western Sydney, Australia, for STI testing, and who had first-void urine (FVU), anorectal and oropharyngeal samples collected, were prospectively enrolled by the attending clinician. M. genitalium testing using the ResistancePlus MG assay was performed on FVU, anorectal and oropharyngeal samples routinely collected for Chlamydia trachomatis and Neisseria gonorrhoeae testing. Men whose tests were positive for $M$. genitalium were treated according to standard clinic protocols, including contact tracing.

The ResistancePlus MG assay (SpeeDx) is a multiplex quantitative (qPCR) assay which uses novel PlexZyme and PlexPrime technology to simultaneously detect $M$. genitalium and five $23 \mathrm{~S}$ rRNA mutations, A2058C, A2058G, A2058T, A2059C and A2059G (Escherichia coli numbering), associated with macrolide resistance. ${ }^{14} 15$ The assay incorporates appropriate positive controls to verify the presence of wild-type M. genitalium (ie, harbouring no resistance mutations) as well as the presence of strains harbouring the above mutations. Two recent studies evaluated this assay and reported excellent sensitivity and specificity for the detection of M. genitalium $(97.4 \%-99.1 \%$ and $98.5 \%-$ $100 \%$, respectively), as well as for the detection of macrolide resistance-associated mutations $\quad(97.4 \%-100 \%$ and $96.2 \%-$ $100 \%$, respectively). ${ }^{14} 15$

Oropharyngeal and anorectal swab specimens consisted of BD ProbeTec dry swabs in diluent (Becton Dickinson, North Ryde, Australia) previously assayed on the BD Viper system (Becton Dickinson) for the routine detection of C. trachomatis and N. gonorrhoeae. Swabs in diluent were vortex mixed for $10 \mathrm{~s}$. DNA was extracted, from either $750 \mu \mathrm{L}$ of mixed swab diluent or $1 \mathrm{~mL}$ of FVU specimens, by on-board lysis workflow on the NucliSens EasyMag platform (bioMerieux Australia, Baulkham Hills, Australia) and eluted in $100 \mu \mathrm{L}$. Five $\mu \mathrm{L}$ of 1 in 10 diluted ResistancePlus MG Internal Control cells (SpeeDx) were added to each specimen prior to lysis addition as described by the manufacturer's evaluation instruction for use document for the manual extraction method.

Real-time detection of M. genitalium by ResistancePlus MG assay (SpeeDx) was performed using the LightCycler 480 Instrument II (Roche Diagnostics Australia, North Ryde, Australia) as described by the manufacturer. A $5 \mu \mathrm{L}$ aliquot of extracted DNA was assayed within a $20 \mu \mathrm{L}$ final reaction volume in 96-well plates using cycling parameters of $95^{\circ} \mathrm{C}$ for $2 \mathrm{~min}$, followed by 10 cycles of $95^{\circ} \mathrm{C}$ for $5 \mathrm{~s}, 61^{\circ} \mathrm{C}-56^{\circ} \mathrm{C}$ for $30 \mathrm{~s}\left(-0.5^{\circ} \mathrm{C}\right.$ per cycle), then 40 cycles of $95^{\circ} \mathrm{C}$ for $5 \mathrm{~s}, 52^{\circ} \mathrm{C}$ for $40 \mathrm{~s}$ and, finally, cooling at $40^{\circ} \mathrm{C}$ for $30 \mathrm{~s}$. Negative assay controls consisted of 5 $\mu \mathrm{L}$ PCR grade water (Sigma-Aldrich, Castle Hill, Australia). Data analysis reporting the presence or absence of M. genitalium, $23 \mathrm{~S}$ rRNA mutation and internal control was performed using the ResistancePlus MG Analysis Software beta version LC480_b2.2 (SpeeDx).

Clinical, demographic and behavioural data were retrospectively collected from laboratory and clinic electronic databases and clinical files at WSSHC. Data recorded included age, number and sex of sexual partners in the last 3 months, condom use, HIV serostatus, use of HIV pre-exposure prophylaxis (PrEP), urethral and/or anorectal symptoms, azithromycin treatment in the last 12 months and co-infection with C. trachomatis and/or N. gonorrhoeae. Urethral symptoms were defined as urethral discharge
Table 1 Characteristics of MSM enrolled $(n=508)$ and not enrolled $(n=47)$

\begin{tabular}{|c|c|c|c|c|c|}
\hline Characteristic & Enrolled & $\begin{array}{l}\text { Not } \\
\text { enrolled }\end{array}$ & OR & $95 \% \mathrm{Cl}$ & $P$ value \\
\hline Mean age (years) & 33.2 & 38.2 & $\mathrm{~N} / \mathrm{A}$ & $\mathrm{N} / \mathrm{A}$ & 0.0030 \\
\hline HIV-positive n (\%) & $30(5.9)$ & $13(27.7)$ & 0.2 & 0.1 to 0.4 & $<0.0001$ \\
\hline CD4 Mean (median) & 775 (791) & 862 (751) & N/A & N/A & 0.3798 \\
\hline On PrEP n (\%) & $169(33.3)$ & $15(31.9)$ & 1.1 & 0.6 to 2.0 & 0.8506 \\
\hline $\begin{array}{l}\text { Urethral symptoms } \\
\mathrm{n}(\%)\end{array}$ & $36(7.1)$ & $2(4.3)$ & 1.7 & 0.4 to 7.4 & 0.4625 \\
\hline $\begin{array}{l}\text { Anorectal symptoms } \\
\mathrm{n}(\%)\end{array}$ & $12(2.4)$ & $1(2.1)$ & 1.1 & 0.1 to 8.8 & 0.9191 \\
\hline $\mathrm{CT} / \mathrm{NG}$ urethral $\mathrm{n}(\%)$ & $25(4.9)$ & $2(4.3)$ & 1.2 & 0.3 to 5.1 & 0.8393 \\
\hline $\begin{array}{l}\text { CT/NG anorectal } \\
\mathrm{n}(\%)\end{array}$ & $67(13.2)$ & $7(15.2)$ & 0.9 & 0.4 to 2.0 & 0.6988 \\
\hline
\end{tabular}

CD4, CD4 cells/ $/ \mathrm{L} ; \mathrm{CT} / \mathrm{NG}$ anorectal, Chlamydia trachomatis and/or Neisseria gonorrhoeae anorectal infection; CT/NG urethral, Chlamydia trachomatis and/or Neisseria gonorrhoeae urethral infection; MSM, men who have sex with men; N/A, not available; PrEP, HIV pre-exposure prophylaxis.

and/or dysuria, and anorectal symptoms were anorectal pain, discomfort, bleeding and/or discharge. Data were entered into Excel and analysed to examine any associations with M. genitalium infection or the presence of macrolide resistance mutations. Prevalence of M. genitalium and macrolide resistance were measured for the study population as a whole, and for specific sites of infection (urethral, anorectal or oropharyngeal).

Data were analysed using Stata Statistical Software: Release 12 (StataCorp, College Station, Texas, USA). Bivariate associations were examined using $X^{2}$ or Fisher's exact tests, and ORs, 95\% CIs and $\mathrm{P}$ values calculated using the Mantel-Haenszel method or multiple logistic regression.

\section{RESULTS}

The study group included 508 consecutive MSM who attended WSSHC from February to May 2017. Enrolled men represented $91.5 \%$ of all eligible men who attended the clinic for STI testing of FVU, anorectal and oropharyngeal samples during the study period. Compared with HIV-negative MSM, HIV-positive MSM were significantly less likely to be enrolled, and were older $(\mathrm{P}=0.002)$. However, there was no significant difference in mean CD 4 cell count, which was $>750$ cells $/ \mu \mathrm{L}$ in HIV-positive MSM enrolled and not enrolled, and rates of urethral or anorectal chlamydial and/or gonococcal infections were similar for MSM with and without HIV infection. Characteristics of men enrolled and not enrolled are detailed in table 1.

\section{Prevalence of $M$. genitalium, macrolide resistance and co- infections}

Overall prevalence of $M$. genitalium infection in the study group was $13.4 \%(68 / 508)$. The anorectum was the most commonly infected site in $45 / 505(8.9 \%)$, followed by the urethra in $24 / 508(4.7 \%)$. There was no result for three men whose anorectal samples were inhibited on testing. No oropharyngeal M. genitalium infections were detected (0/508). Only one man had both urethral and anorectal $M$. genitalium infection. Among asymptomatic men, the prevalence of M. genitalium was $8.5 \%(42 / 493)$ in the anorectum, and $4.0 \%(19 / 472)$ in the urethra. Overall, $79.4 \%(54 / 68)$ of infections were macrolide-resistant, with 21/24 (87.5\%) of urethral and $34 / 45(75.6 \%)$ of anorectal infections harbouring macrolide resistance-associated mutations 


\begin{tabular}{|c|c|c|c|c|}
\hline & $\begin{array}{l}\text { MG positive } n \\
(\%)\end{array}$ & $\begin{array}{l}\text { MG negative } n \\
(\%)\end{array}$ & OR $(95 \% \mathrm{Cl})$ & $P$ value \\
\hline $\begin{array}{l}\text { Age group } \\
\text { (years) }\end{array}$ & & & $0.9(0.7$ to 1.2$)$ & 0.4261 \\
\hline$\leq 29$ & 34 (13.4) & $219(86.6)$ & 0.2 (0.1 to 0.2$)$ & \\
\hline 30-39 & $20(14.1)$ & $122(85.9)$ & 0.2 (0.1 to 0.3 ) & \\
\hline 40-49 & $12(19.4)$ & $50(80.7)$ & 0.2 (0.1 to 0.5$)$ & \\
\hline$\geq 50$ & $2(3.9)$ & $49(96.1)$ & $0.0(0.0$ to 0.2$)$ & \\
\hline On PrEP & & & 2.1 (1.3 to 3.6 ) & 0.0041 \\
\hline Yes & $33 / 169$ (19.5) & $136 / 169(80.5)$ & & \\
\hline No & 35/339 (10.3) & $304 / 339(89.7)$ & & \\
\hline Anorectal CT & & & 3.9 (1.8 to 8.5$)$ & 0.0002 \\
\hline Yes & $11 / 45$ (24.4) & $35 / 460(7.6)$ & & \\
\hline No & $34 / 45$ (75.6) & $425 / 460(92.4)$ & & \\
\hline Anorectal NG & & & 1.5 (0.5 to 4.3$)$ & 0.5036 \\
\hline Yes & $4 / 45(8.9)$ & $29 / 460(6.3)$ & & \\
\hline No & $41 / 45$ (91.1) & $431 / 460$ (93.7) & & \\
\hline Urethral CT & & & $2.2(0.5$ to 10.2$)$ & 0.2902 \\
\hline Yes & $2 / 24(8.3)$ & 19/484 (3.9) & & \\
\hline No & 22/24 (91.7) & $465 / 484$ (96.1) & & \\
\hline Urethral NG & & & 3.0 (0.4 to 25.2$)$ & 0.2966 \\
\hline Yes & $1 / 24(4.2)$ & $7 / 484(1.5)$ & & \\
\hline No & 23/24 (95.8) & 477/484 (98.6) & & \\
\hline $\begin{array}{l}\text { Male partners } \\
\text { last } 3 / 12\end{array}$ & & & $1.2(0.9$ to 1.5$)$ & 0.30 \\
\hline 0 & $1 / 8(12.5)$ & $7 / 8(87.5)$ & 0.1 & \\
\hline 1 & $12 / 86(14.0)$ & 74/86 (86.1) & 0.2 & \\
\hline $2-5$ & 29/252 (11.5) & $223 / 252(88.5)$ & 0.1 & \\
\hline $6-10$ & 16/96 (16.7) & 80/96 (83.3) & 0.2 & \\
\hline$>10$ & 10/57 (17.5) & $47 / 57$ (82.5) & 0.2 & \\
\hline $\begin{array}{l}\text { Female partners } \\
\text { last } 3 / 12\end{array}$ & & & $1.8(0.8$ to 4.2$)$ & 0.1511 \\
\hline Yes & $8 / 68(11.8)$ & $30 / 439$ (6.8) & & \\
\hline No & 60/68 (88.2) & 409/439 (93.2) & & \\
\hline Condom use & & & 0.8 (0.6 to 1.0$)$ & 0.0186 \\
\hline Never & 16/94 (17.0) & 78/94 (83.0) & 0.2 & \\
\hline$<50 \%$ & 19/117 (16.2) & 98/117 (83.8) & 0.2 & \\
\hline$\geq 50 \%$ & 24/168 (14.3) & $144 / 168(85.7)$ & 0.2 & \\
\hline Always & $7 / 108$ (6.5) & $101 / 108(93.5)$ & 0.1 & \\
\hline N/A & $1 / 15(6.7)$ & 14/15 (93.3) & 0.1 & \\
\hline HIV infection & & & 0.7 (0.2 to 2.4$)$ & 0.5749 \\
\hline Yes & $3 / 30(10.0)$ & $27 / 30(90.0)$ & & \\
\hline No & 65/478 (13.6) & $413 / 478(86.4)$ & & \\
\hline
\end{tabular}

Condom use, condom use for anal sex last 3 months: N/A, no anal sex in the last 3 months (data missing for six men); female partners last 3/12, any female sexual partner/s in the last 3 months (data missing for one man); male partners last 3/12, number of male sexual partners in the last 3 months (data missing for nine men). $\mathrm{CT}$, Chlamydia trachomatis; MG, Mycoplasma genitalium; N/A, not available; NG, Neisseria gonorrhoeae; PrEP, HIV pre-exposure prophylaxis.

(no significant difference; $\mathrm{P}=0.25$ ). Apart from being less common among men aged 50 years and older, presence of $M$. genitalium infection was not related to age (table 2). In this cohort, C. trachomatis was detected in 65/508 (12.8\%) men overall, in $21 / 508(4.1 \%)$ FVU samples, in $46 / 508(9.1 \%)$ anorectal samples and in 10/508 (2.0\%) oropharyngeal samples. Likewise, N. gonorrhoeae were detected in $62 / 508$ (12.2\%) overall, in 8/508 (1.6\%) FVU samples, in 33/508 $(6.5 \%)$ anorectal samples and in $43 / 508$ (8.5\%) oropharyngeal samples.
Association between macrolide resistance and previous azithromycin treatment

Information on azithromycin treatment in the last 12 months was available for $65 / 68$ of the men with M. genitalium infection. Among 52 men with macrolide-resistant strains, 46.2\% had been treated with azithromycin within 12 months. While $24 / 25$ (96.0\%) of men who had received azithromycin had macrolide-resistant M. genitalium infection, 28/40 (70.0\%) of those who had not also harboured resistant strains. Men who had received azithromycin treatment in the last 12 months were significantly more likely to have a macrolide-resistant $M$. genitalium infection (OR 10.3, 95\% CI 1.1 to 96.9, $\mathrm{P}=0.0114$ ). No other study factor was associated with presence of macrolide-resistance mutations in M. genitalium, including presence of urethral $(\mathrm{P}=0.9606)$ or anorectal $(\mathrm{P}=0.3706)$ symptoms.

\section{Associations between M. genitalium, STI co-infections, HIV and symptoms}

Associations between M. genitalium, symptoms and other infections are presented in table 2. Co-infections with C. trachomatis were more common than with $N$. gonorrhoeae. Two men had anorectal infection with all three microorganisms. Anorectal C. trachomatis was independently associated with anorectal M. genitalium (OR 5.0, 95\% CI 2.1 to $11.8, \mathrm{P}<0.001$ ) after controlling for condom use, number of male sexual partners in the last 3 months, age, anorectal N. gonorrhoeae, use of PrEP and HIV infection. HIV-infected men were not more likely than men without HIV infection to test positive for M. genitalium or to have infection with C. trachomatis or $N$. gonorrhoeae $(\mathrm{P}=0.26)$.

M. genitalium was detected in 3 of the 12 men with anorectal symptoms, of whom one had co-infection with C. trachomatis. Very few anorectal infections were associated with symptoms, and a consistently significant association was found only for anorectal gonococcal infection (table 3). Urethral gonococcal or chlamydial co-infection was detected in two of the five men with urethral symptoms who tested positive for M. genitalium. For all three pathogens, urethral infection was independently associated with presence of urethral symptoms (table 3).

\section{Associations with PrEP, partners and condom use}

Men who were on PrEP were twice as likely to be infected with M. genitalium compared with men who were not on PrEP (table 2). There was no difference in the prevalence of macrolide resistance-associated mutations in $M$. genitalium infections among men on PrEP (78.8\%) compared with men not on PrEP (80.0\%), OR 0.9 (95\% CI 0.3 to 3.0, P=0.9024). The number of recent male sexual partners did not predict M. genitalium positivity; however, always using condoms for anal sex in the last 3 months was protective of infection (table 2). Men who always used condoms for anal sex in the last 3 months were less likely to be on PrEP (OR 0.6, 95\% CI 0.5 to $0.7, \mathrm{P}<0.001$ ). On multivariate analysis, controlling for condom use, number of male partners in the last 3 months, age, other urethral or anal infections and HIV infection, M. genitalium positivity remained significantly associated with use of PrEP (OR 2.5, 95\% CI 2.0 to 5.2, $\mathrm{P}=0.015)$.

\section{DISCUSSION}

Key findings of our study were the high frequency of $M$. genitalium infection and very high prevalence of M. genitalium macrolide resistance in MSM attending our clinic in western Sydney. Our study also confirms that the oropharynx is not an important site for M. genitalium infection. 
Table 3 Associations between anogenital symptoms and STIs

\begin{tabular}{|c|c|c|c|c|}
\hline Site of infection & Pathogen & Symptoms* n (\%) & OR $(95 \% \mathrm{Cl})$ & aOR $(95 \% \mathrm{Cl})$ \\
\hline \multirow[t]{3}{*}{ Anorectal } & Mycoplasma genitalium & $3 / 45(6.7)$ & $3.6(0.9$ to 13.8$)$ & 4.0 (1.0 to 16.3$)$ \\
\hline & Chlamydia trachomatis & $1 / 46(2.2)$ & $0.9(0.1$ to 7.2$)$ & 0.3 (0.0 to 2.8 ) \\
\hline & Neisseria gonorrhoeae & $4 / 33(12.1)$ & 8.1 (2.3 to 28.9$)$ & 10.2 (2.7 to 39.1$)$ \\
\hline \multirow[t]{3}{*}{ Urethral } & M. genitalium & $5 / 24(20.8)$ & $3.9(1.3$ to 11.1$)$ & 3.5 (1.0 to 11.8$)$ \\
\hline & C. trachomatis & $12 / 21(57.1)$ & 25.7 (9.0 to 73.3$)$ & $19.0(6.7$ to 53.4$)$ \\
\hline & N. gonorrhoeae & $6 / 8(75.0)$ & 47.0 (8.2 to 269.0$)$ & 21.7 (3.3 to 143.1$)$ \\
\hline
\end{tabular}

*Anorectal symptoms in the case of anorectal infection, urethral symptoms in the case of urethral infection.

$\mathrm{aOR}$, adjusted $\mathrm{OR}$ after controlling for the other two anorectal or urethral infections.

Almost one in eight men was infected with M. genitalium, most commonly in the anorectum. In this cohort, the overall prevalence of M. genitalium was similar to that of both C. trachomatis and N. gonorrhoeae. More than three-quarters of all urethral and anorectal M. genitalium infections harboured macrolide resistance-associated mutations. We used a new multiplex qPCR assay, ResistancePlus MG (SpeeDx), that simultaneously detects the presence of M. genitalium and macrolide resistance-associated mutations. ${ }^{14} 15$ A realistic laboratory procedure turnaround time for this assay is up to 72 hours from laboratory receipt of the sample. Results can be available within five working days of sample collection, enabling initial treatment with moxifloxacin for men with macrolide-resistant $M$. genitalium infection, and facilitating appropriate management of sexual contacts. While moxifloxacin is likely to be effective in the majority of these cases, further treatment options for men whose M. genitalium infections are also resistant to moxifloxacin remain incompletely evaluated. $^{13}$

Macrolide resistance in anorectal infections has not previously been systematically investigated, but was recently detected in 7/7 $(100 \%)$ anorectal samples in Melbourne, Australia. ${ }^{15}$ Selection of macrolide-resistant strains is likely among groups with high rates of partner change and frequent episodes of STI treatment. In our study, presence of macrolide resistance in M. genitalium strains was associated with azithromycin treatment in the last 12 months, and this finding was also recently reported by investigators in Spain. ${ }^{16}$ Although we now routinely use doxycycline for initial treatment of NGU and anorectal chlamydial infection, azithromycin is still used in combination with ceftriaxone for the treatment of gonococcal infections. Nevertheless, there was no history of prior azithromycin in over half of the men with macrolide-resistant infections, suggesting that resistant infections are being commonly transmitted and acquired.

Only a small proportion of both urethral and anorectal infections were symptomatic in this cohort, providing some information regarding the symptomatic proportion of $M$. genitalium infections among MSM; only one-fifth of MSM with urethral, and $6.7 \%$ with anorectal M. genitalium infections were symptomatic. However, it is difficult to determine if the presence of symptoms was due to M. genitalium in all cases as another pathogen was detected in almost half of urethral and in a third of anorectal symptomatic infections.

There is currently no international consensus on screening of asymptomatic MSM for M. genitalium. Among high-risk MSM, asymptomatic anorectal $M$. genitalium represents a reservoir of transmissible infection, while antibiotic treatment for other STIs is probably contributing to rising M. genitalium antimicrobial resistance. Consequently, it is likely that transmission of macrolide-resistant infections will continue unchecked. Nevertheless, the public health value of testing asymptomatic men for M. genitalium has not been established.
Although recent European guidelines advise that regular $M$. genitalium testing including anal sampling could be considered due to the risk of increased HIV transmission, we lack evidence of synergy between M. genitalium and HIV in asymptomatic MSM, as the majority of data supporting this interaction relate to women ${ }^{77-20}$ Additionally, there is only limited evidence that M. genitalium causes proctitis. No significant association with anorectal symptoms was found in three observational studies. ${ }^{1-3}$ However, in one study of 154 men with proctitis, 12\% tested positive for M. genitalium, with significantly higher organism loads among cases compared with men with asymptomatic rectal infection. ${ }^{21}$ Stronger evidence implicating M. genitalium in proctitis and/or HIV transmission/acquisition in MSM would support expanding M. genitalium screening for asymptomatic MSM. However, we still lack clarity on whether or not all M. genitalium strains are pathogenic, which makes interpretation of positive screening test results difficult in the absence of symptoms or disease. Of more practical importance, the lack of reliable treatment alternatives in cases where moxifloxacin treatment fails, and the level of patient anxiety that ensues, must also be considered in the screening risk-benefit equation.

The increased prevalence of M. genitalium infection in men taking PrEP is largely but not entirely explained by lower rates of condom use. In this cohort, M. genitalium infection was not associated with HIV infection per se. Several previous studies have reported that $M$. genitalium was more common in MSM who were HIV-positive compared with those HIV-negative. $^{3}{ }^{21}$ Reasons underlying differences between studies may reflect behavioural factors, the degree of HIV-associated immunosuppression and density of sexual networks.

Our study had several limitations. First, some eligible men, particularly HIV-positive patients, were not recruited due to clinicians failing to enrol them. However, analysis of recruited patients showed that there was no difference in prevalence of M. genitalium infection related to HIV status, and comparison of HIV-positive men enrolled and not enrolled did not show any difference in prevalence of other STIs. Secondly, the sample size was designed to measure prevalence of infection, but may not have been large enough to accurately measure the proportion of symptomatic M. genitalium infection. Thirdly, use of azithromycin in the last 12 months may have been underestimated if men had received azithromycin elsewhere and that was not recorded in the clinical files. Lastly, the study was conducted in a single clinic and results may not be applicable to other clinical settings in Australia or internationally.

In conclusion, we have demonstrated a high prevalence of $M$. genitalium among MSM attending our service, and highlighted therapeutic challenges posed by high levels of macrolide resistance. By prolonging duration of infection and infectivity, antimicrobial resistance-related treatment failure is likely implicated in rising M. genitalium prevalence. Our findings support the 
routine use of an assay to both detect $M$. genitalium infection as well as identify the presence of any macrolide resistance mutations, as recommended in the recent European guidelines on $M$. genitalium. ${ }^{7}$ Such an assay could ensure that first-line treatment with azithromycin is only used if a macrolide-sensitive M. genitalium strain is identified in regions or populations with high rates of macrolide resistance. Finally, this new diagnostic tool could assist with ongoing efforts to strengthen M. genitalium antimicrobial resistance surveillance as well as inform local and regional M. genitalium treatment guidelines.

\section{Key messages}

- $13.4 \%$ of men who have sex with men in western Sydney have Mycoplasma genitalium infection, most commonly anorectal (8.9\%), followed by urethral $(4.7 \%)$; no oropharyngeal infections were detected.

- Most anorectal (93.3\%) and urethral (79.2\%) infections were asymptomatic.

- Nearly $80 \%$ of $M$. genitalium infections harboured macrolide resistance-associated mutations.

- A diagnostic assay that can simultaneously detect $M$. genitalium and macrolide resistance-associated mutations will help to limit inappropriate azithromycin treatment.

\section{Handling editor Catherine A Ison}

Acknowledgements The authors acknowledged the assistance from SpeeDx

Contributors All of the authors contributed to study design. MP, DLC and DAL planned and conducted the clinical aspects, and DJ, NJJ and SC planned and conducted the laboratory aspects, of the study. DJ performed most of the laboratory assays and laboratory data collection, and NJJ and SC had oversight of laboratory procedures. DLC collected clinical data, performed statistical analyses and wrote the initial manuscript draft, apart from laboratory procedures, which were written by DJ. All of the authors reviewed and made contributions to the final manuscript.

Funding This research received no specific grant from any funding agency in the public, commercial or not-for-profit sectors. SpeeDx (SpeeDx Pty Ltd, Sydney, NSW, Australia) is the developer and manufacturer of the assay used in this study, and supplied the ResistancePlus MG assay study test kits free of charge.

Competing interests SpeeDx personnel assisted with specimen extraction and provided laboratory technical advice, but were not involved in performing the ResistancePlus MG assays, or in the design, conduct or reporting of this study.

Ethics approval This study was approved by Western Sydney Local Health District Human Research Ethics Committee, HREC reference number: HREC/16/WMEAD/280; SSA reference number SSA/16/WMEAD/304.

Provenance and peer review Not commissioned; externally peer reviewed.

(c) Article author(s) (or their employer(s) unless otherwise stated in the text of the article) 2018. All rights reserved. No commercial use is permitted unless otherwise expressly granted.

\section{REFERENCES}

1 Francis SC, Kent CK, Klausner JD, et al. Prevalence of rectal Trichomonas vaginalis and Mycoplasma genitalium in male patients at the San Francisco STD clinic, 2005-2006. Sex Transm Dis 2008;35:797-800.

2 Bradshaw CS, Fairley CK, Lister NA, et al. Mycoplasma genitalium in men who have sex with men at male-only saunas. Sex Transm Infect 2009;85:432-5.

3 Soni S, Alexander S, Verlander N, et al. The prevalence of urethral and rectal Mycoplasma genitalium and its associations in men who have sex with men attending a genitourinary medicine clinic. Sex Transm Infect 2010;86:21-4.

4 Reinton N, Moi H, Olsen AO, et al. Anatomic distribution of Neisseria gonorrhoeae, Chlamydia trachomatis and Mycoplasma genitalium infections in men who have sex with men. Sex Health 2013;10:199-203.

5 Zheng BJ, Yin YP, Han Y, et al. The prevalence of urethral and rectal Mycoplasma genitalium among men who have sex with men in China, a cross-sectional study. BMC Public Health 2014;14:195-201.

6 Fuchs W, Kreuter A, Hellmich M, et al. Asymptomatic anal sexually transmitted infections in HIV-positive men attending anal cancer screening. Br J Dermatol 2016;174:831-8.

7 Jensen JS, Cusini M, Gomberg M, et al. 2016 European guideline on Mycoplasma genitalium infections. J Eur Acad Dermatol Venereol 2016;30:1650-6.

8 Manhart LE. Mycoplasma genitalium on the loose: time to sound the alarm. Sex Transm Dis 2017;44:463-5.

9 Edlund M, Blaxhult A, Bratt G. The spread of Mycoplasma genitalium among men who have sex with men. Int J STD AIDS 2012;23:455-6.

10 Jensen JS, Bradshaw C. Management of Mycoplasma genitalium infections - can we hit a moving target? BMC Infect Dis 2015;15:343.

11 Couldwell DL, Lewis DA. Mycoplasma genitalium infection: current treatment options, therapeutic failure, and resistance-associated mutations. Infect Drug Resist 2015;8:147-61.

12 Tagg KA, Jeoffreys NJ, Couldwell DL, et al. Fluoroquinolone and macrolide resistanceassociated mutations in Mycoplasma genitalium. J Clin Microbiol 2013;51:2245-9.

13 Bissessor M, Tabrizi SN, Twin J, et al. Macrolide resistance and azithromycin failure in a Mycoplasma genitalium-infected cohort and response of azithromycin failures to alternative antibiotic regimens. Clin Infect Dis 2015;60:1228-36.

14 Tabrizi SN, Tan LY, Walker S, et al. Multiplex assay for simultaneous detection of Mycoplasma genitalium and macrolide resistance using PlexZyme and PlexPrime technology. PLoS One 2016;11:e0156740.

15 Tabrizi SN, Su J, Bradshaw CS, et al. Prospective evaluation of ResistancePlus MG, a new multiplex quantitative PCR assay for detection of Mycoplasma genitalium and macrolide resistance. J Clin Microbiol 2017;55:1915-9.

16 Barberá MJ, Fernández-Huerta M, Jensen JS, et al. Mycoplasma genitalium macrolide and fluoroquinolone resistance: prevalence and risk factors among a 2013-2014 cohort of patients in Barcelona, Spain. Sex Transm Dis 2017;44:457-62.

17 Napierala Mavedzenge S, Weiss HA. Association of Mycoplasma genitalium and HIV infection: a systematic review and meta-analysis. AIDS 2009;23:611-20.

18 Napierala Mavedzenge S, Müller EE, Lewis DA, et al. Mycoplasma genitalium is associated with increased genital HIV type 1 RNA in Zimbabwean women. $J$ Infect Dis 2015;211:1388-98.

19 Vandepitte J, Weiss HA, Bukenya J, et al. Alcohol use, Mycoplasma genitalium, and other STIs associated with HIV incidence among women at high risk in Kampala, Uganda. J Acquir Immune Defic Syndr 2013;62:119-26.

20 Vandepitte J, Weiss HA, Bukenya J, et al. Association between Mycoplasma genitalium infection and HIV acquisition among female sex workers in Uganda: evidence from a nested case-control study. Sex Transm Infect 2014;90:545-9.

21 Bissessor M, Tabrizi SN, Bradshaw CS, et al. The contribution of Mycoplasma genitalium to the aetiology of sexually acquired infectious proctitis in men who have sex with men. Clin Microbiol Infect 2016;22:260-5. 